\title{
BIODIVERSITY OF TERRESTRIAL MALACOCOENOSES AS A CRITERION IN ASSESSMENT OF CONSERVATION STATUS - THE CYBINA VALLEY, A PROPOSED NATURA 2000 AREA
}

\author{
ELŻBIETA KORALEWSKA-BATURA, JERZY BŁOSZYK, AGNIESZKA NAPIERAŁA, BARTŁOMIEJ GOŁDYN
}

Department of General Zoology, Institute of Environmental Biology, Faculty of Biology, Adam Mickiewicz University, Umultowska 89, 61-614 Poznań, Poland (e-mail: bloszyk@amu.edu.pl)

ABSTRACT: We present the results of a terrestrial mollusc inventory in the Cybina River Valley (W. Poland). The species richness in 39 sites served as a biodiversity criterion for the whole area. The total number of 3,193 specimens collected represented 51 species (22 families), i.e. about $60 \%$ of terrestrial snail species of the whole Wielkopolska region. Species regarded as rare in the region and in the whole country constituted $22 \%$ of the whole material. Two of them are especially noteworthy: Vertigo angustior Jeffreys (Annex II, EU Habitats Directive), and Ruthenica filograna (Rossmässler) which is very rare in the region.

KEY WORDS: biodiversity, terrestrial gastropods, river valley, Poland, Natura 2000

\section{INTRODUCTION}

The international Convention on Biological Diversity, signed (5.06.1992) and ratified (18.01.1996) by Poland, obliges the state authorities to protect the biodiversity of ecosystems, habitats and species, reduce the impact leading to decline in habitats and preserve the populations in their natural environment (UNO 1992). Moreover, changes in the natural resources have to be monitored and assessed. Thus, intensification of inventory studies is now even more essential than before. On the other hand, the lack of proper documentation of conservation value of some areas makes it impossible to protect them under any legal status and to form appropriate land development plans.

According to the map of natural vegetation of central Poland (WOJTERSKI et al. 1978), in the past most of western and central Wielkopolska was covered by deciduous forests with oak-hornbeam formations prevailing, with some coniferous forests, while the river valleys held riparian forests. Deforested areas were in negligible in the region. At present, forests occupy only $25.5 \%$ of the area, and the landscape is mostly agricultural. Though most indigenous forest species of plants and animals have survived, the progressive deforestation and simplification of the landscape have depleted their habitats, making their further survival uncertain. Some, being unable to resist the pressure, have already become extinct or very rare and at present occur only in vestigial fragments of their natural habitats; some have been able to colonise other, human-transformed habitats.

Valleys of small rivers provide an optimal spatio-functional layout for studies on environmental changes in the North European Plain. Such areas usually have all the characteristic landscape components; the ecological processes there influence the conservation value of larger geographical units of which these areas form a part. The aim of this study was to assess the species composition of terrestrial malacocoenoses in the Cybina River Valley and to evaluate the mollusc diversity in relation to the diversity and fragmentation of habitats. 


\section{MATERIAL AND METHODS}

The study was conducted in 2004-2005 in the Cybina River Valley, a right-bank tributary of the Warta River. The valley is located in the Wielkopolska Lakeland macroregion, central-western Poland. The study covered the whole course of the river, from its source in the vicinity of Nekielka village $\left(52^{\circ} 23^{\prime} 38^{\prime \prime} \mathrm{N}\right.$, $\left.17^{\circ} 22^{\prime} 00^{\prime \prime} \mathrm{E}\right)$ to its estuary in Poznań, where the Cybina joins the Warta River $\left(52^{\circ} 24^{\prime} 35^{\prime \prime} \mathrm{N}, 16^{\circ} 57^{\prime} 06^{\prime} \mathrm{E}\right)$ (Fig. 1).

Malacofauna was sampled in 42 selected plots covering fragments of the valley bottom and slopes, as well as fragments of adjacent moraine height. The areas omitted in the present study (1-3, situated within the borders of the city of Poznań) covered localities with small habitat diversity, much modified under human impact. Each plot was $1 \mathrm{~km}$ long; the plots were numbered according to their position along the river, from the sources to the mouth (GOŁDYN \& GRABIA 1998, GOŁDYN et al. 2005). The sampling effort varied among the plots according to their habitat diversity. We used two collecting methods. Quantitative samples were taken with Oekland frame of $25 \times 25 \mathrm{~cm}$; litter and ca. $5 \mathrm{~cm}$ soil layer were collected. The samples were hand-sorted in the laboratory. In total, 117 such samples were collected (3 samples from each plot) and snails were found in 66 of them (56\% of the samples). Gastropods were also recorded by eye, usually after a rain or early in the morning. In case of large and abundant species, we collected only single individuals; the total abundance and distribution were recorded.

The nomenclature follows PIECHOCKI \& SULIKOWSKA-DROZD (2008). The material is deposited in the Natural History Collections of the Faculty of Biology, Adam Mickiewicz University, Poznań.

Similarity of snail communities between particular areas was calculated with the Marczewski-Steinhaus index of species similarity (HOLGATE 1969). The nearest neighbour cluster analysis method with the Bray-Curtis similarity measure was used (EvERITT et al. 2001). Coefficients of dominance (D\%) and constancy $(\mathrm{C} \%)$ were calculated for positive samples $(\mathrm{n}=66)$. The dominance and constancy classes were applied after BŁOsZYK (1999): Dominance (D\%): D5 - eudominants $>30.0 \%$, D 4 - dominants $15.1-30.0 \%$, D3 - subdominants $7.1-15.0 \%$, D2 - recedents 3. 0-7.0\%, D1 - subrecedents < 3\%; Constancy (C\%): C5 - euconstants $>50 \%$, C4 - constants $30.1-50.0 \%$, C3 - subconstants 15.1-30.0\%, C2 - accessory species $5.0-15.0 \%, \mathrm{C} 1-$ accidental species $<5.0 \%$. The species were classified in four frequency categories with respect to the number of plots $(n=39)$ where they were collected: very common found in more than $51 \%$ of the plots; common $-31-50 \%$; rare $-10-30 \%$; very rare - less than $10 \%$.

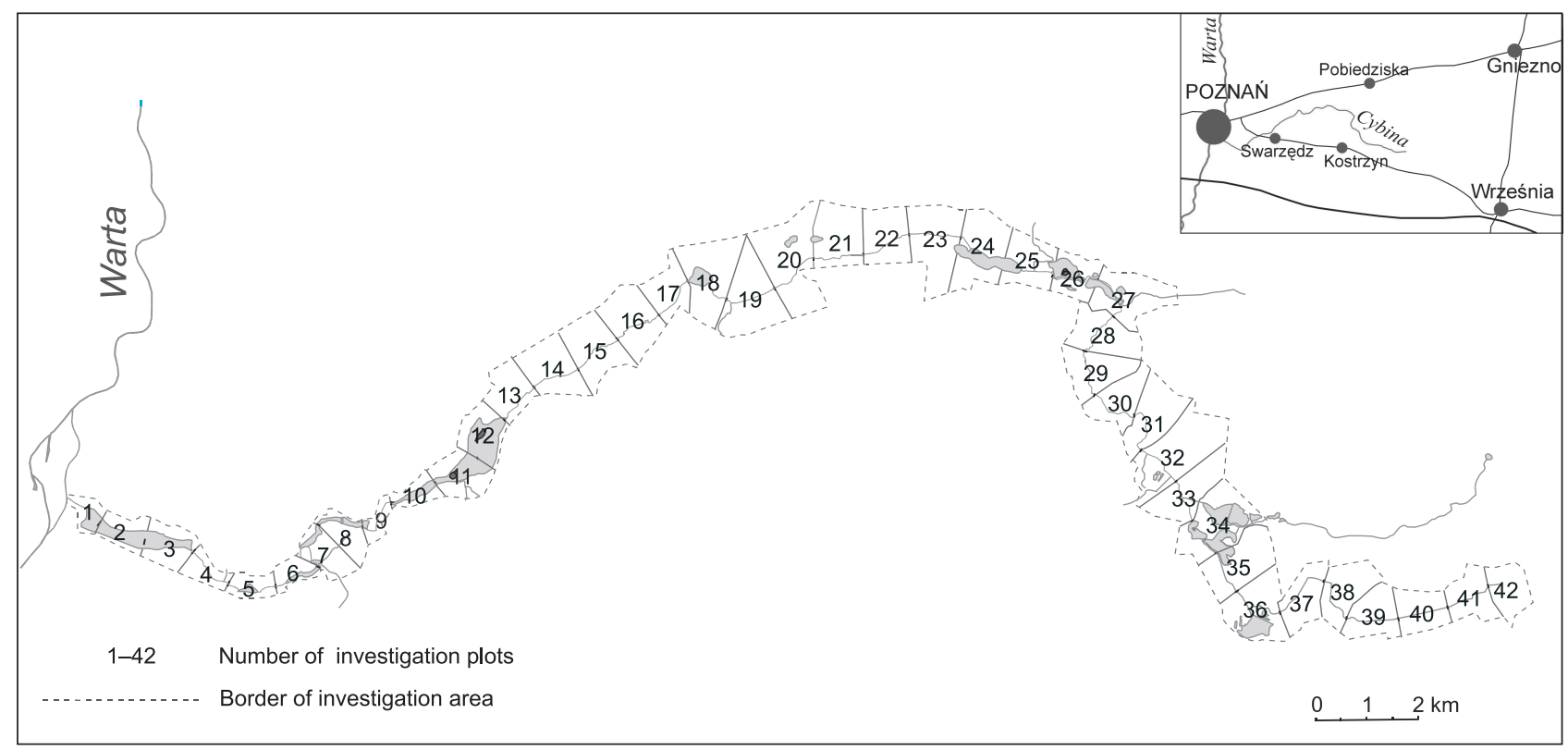

Fig. 1. Cybina Valley - investigated plots 


\section{RESULTS}

\section{COMPLETENESS OF THE RESULTS}

Out of the 26 plots which yielded any specimens, only 10 met the criterion "ten times as many specimens as there are species" (CAMERON \& POKRYSZKO 2005) (see numbers of species and specimens in Table 1). The values of the Chao index (CAMERON \& POKRYSZKO 2005) for individual plots varied from 0 to 18 (mean $\approx 5)$, the value for all the study area was 10.13, indicating that a few species are missing from most plots, while species not recorded in this study can be expected to be found in the valley.

\section{COMPOSITION, RICHNESS, STRUCTURE AND DIVERSITY OF THE MALACOCOENOSES}

Gastropods were found in 26 out of the 39 examined plots $(66 \%)$. The total number of 3,193 specimens was collected; they represented 51 species of 22 families (Table 1). The most numerous species were Helix pomatia, Arianta arbustorum, Cochlicopa lubrica, Cepea nemoralis and Nesovitrea hammonis, all classified as subdominants. The relatively high participation of $R u$ thenica filograna (one of the recedents) is noteworthy since the species is very rare in the region (KORALEWSKA-BATURA 1992, KORALEWSKA-BATURA et al. 2010). The participation of particular species in the samples was very similar. No species was a clear dominant in the communities (Table 2), which is frequently observed among different groups of soil fauna when the stability of the soil environment is disturbed (BŁOSZYK et al. 2006). The most constant species recorded in the samples was $N$. hammonis, an euconstant found in more than half of the samples. It was followed by constants: C. lubrica, H. pomatia, Trichia hispida, Vallonia costata and V. pulchella, with constancy between 33 and 48\%. Accidental species were relatively numerous in the material (22) (Table 2).

No species could be regarded as very common according to the used frequency scale; no species was recorded from more than $20(51 \%)$ plots. Seven species were common: $H$. pomatia and $N$. hammonis (19 plots each), C. lubrica (18), V. costata and T. hispida (16 each), V. pulchella (14), Zonitoides nitidus (13) and Carychium minimum (12). Rare species included Punctum pygmaeum (11 plots), Succinea putris, Cepaea nemoralis and Euomphalia strigella (10 each), Cochlicopa lubricella, Pseudotrichia rubiginosa and A. arbustorum (9 each), Perforatella bidentata and Monachoides incarnatus (7 each), Euconulus fulvus and Succinella oblonga (6 each). Five species were noticed from five plots each (Vertigo pusilla, Merdigera obscura, Arion fuscus, Nesovitrea petronella and Fruticicola fruticum) and six from four plots each (Truncatellina costulata, Vertigo angustior, Vitrina pellucida, Vitrea crystallina, Aegopinella nitidula and Ae. pura). Twenty one species were very rare in the Cybina Valley. Nine of them occurred in only one plot each (Platyla polita, Oxyloma elegans, Cochlicopa nitens, Vertigo antivertigo, Acanthinula aculeata, Oxychilus draparnaudi, Limax maximus, Deroceras agreste, D. laeve), eight - in two plots each (Truncatellina cylindrica, Vertigo pygmaea, V. substriata, Pupilla muscorum, Chondrula tridens, Discus rotundatus, Deroceras reticulatum and Xerolenta obvia) and four in three plots each (Cepaea hortensis, Clausilia bidentata, Columella edentula and R. filograna).

Several plots were found to hold from zero to 42 species. The species richness was greatest in plot 4 (riverine forest between the Malta reservoir and the Olszak pond). Three plots had more than 20 species: plot 19 (meadows and riverine forest between Lake Uzarzewskie and Janikowo village) with 26 species, plot 25 (meadows between Lake Góra and the pond complex in Promno) with 25 species, and plot 33 (meadows and riverine forest $1 \mathrm{~km}$ downstream from Iwno) with 21 species. Eleven plots $(12,15,16,18,21$, $27,33,34,35,37,41)$ held from 10 to 18 species. The number of species in each of the remaining plots was smaller than 10. No snails were found in the samples collected from 13 plots (numbers $6,7,9,13,14,20$, 22, 23, 24, 29, 30, 31 and 32).

According to species composition (Fig. 2), plots 19 and 4 were the most similar $(\mathrm{S}=62 \%)$ (the only plots with $V$. pygmaea, P. muscorum, D. reticulatum and $X$. obvia, with $R$. filograna and $V$. angustior and the largest total number of species), as well as plots 41 and 16 , and 37 and 36 (in case of both pairs, the most abundant populations of the most common species in the valley were present, with large proportion of $H$. pomatia in plots 37 and 38). Plots 26 and 17, with the fewest species, were the most distinct. However, all the similarities were low (mean $\mathrm{S}=22 \%$ ), indicating considerable differences among the snail communities in the Cybina Valley, most probably resulting from the variety of available habitats.

\section{DISCUSSION}

This study is a result of a preliminary inventory of the terrestrial malacofauna of the Cybina Valley. More detailed research in some of the plots will surely reveal more numerous species which is also indicated by the values of the Chao index. Such research, based on regular samples taken during a longer period of time, should be regarded as high priority. The catchment area of the Cybina River is located in intensively 


\begin{tabular}{|c|c|c|c|c|c|c|c|c|c|c|c|c|c|c|c|c|c|}
\hline \multicolumn{2}{|c|}{ 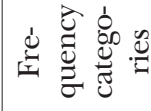 } & $\stackrel{f}{s}$ & U & $\simeq$ & $\simeq$ & 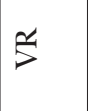 & U & $\simeq$ & $\stackrel{s}{5}$ & $\stackrel{s}{5}$ & $\simeq$ & $\stackrel{f}{5}$ & $\stackrel{f r}{5}$ & $\simeq$ & $\stackrel{f r}{s}$ & $\stackrel{f}{\xi}$ & $\simeq$ \\
\hline \multicolumn{2}{|c|}{ 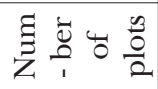 } & - & $\stackrel{\mathcal{V}}{\sim}$ & 0 & 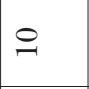 & - & $\underset{-\infty}{-\infty}$ & 0 & - & on & $r$ & or & - & 10 & ov & or & 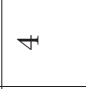 \\
\hline \multicolumn{2}{|c|}{ 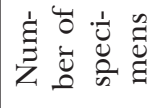 } & - & $\stackrel{\cong}{\exists}$ & $\stackrel{20}{2}$ & in & - & $\begin{array}{l}\infty \\
\stackrel{1}{2}\end{array}$ & $\mathscr{F}$ & 0 & $\stackrel{20}{2}$ & $\stackrel{\Delta}{\sim}$ & $\infty$ & or & $\stackrel{\sim}{\sim}$ & 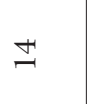 & $\underline{\sim}$ & $\exists$ \\
\hline \multirow{11}{*}{$\mid \begin{array}{l}\overrightarrow{0} \\
\frac{0}{2} \\
\frac{g}{u} \\
\tilde{d}\end{array}$} & Y & & $\infty$ & & - & & $\infty$ & & & & & & & & & & \\
\hline & $F$ & & ov & - & - & & 10 & & & & & & & & & & \\
\hline & q & & & - & & & & & & & & & & & & & \\
\hline & கి & & ov & & & & & $\infty$ & & & & & & & & & \\
\hline & $\infty$ & & & & & & 1 & & & & & & & & & & \\
\hline & 命 & & & & & & & $\infty$ & & & & & & & & & \\
\hline & क & & & & & & $\infty$ & on & & & $\stackrel{\mathcal{Y}}{\sim}$ & - & & & & & \\
\hline & 10 & & & - & & & $H$ & $\simeq$ & & & $\infty$ & & & & & & \\
\hline & के & & - & & & & $\infty$ & 0 & & & & & & - & & & \\
\hline & $\stackrel{m}{m}$ & & $\sigma$ & 20 & & & $\infty$ & $\infty$ & & & - & & & - & & $\stackrel{2}{2}$ & \\
\hline & $\stackrel{\infty}{\sim}$ & & - & & & & ov & & & & & & & & & & \\
\hline \multirow{7}{*}{ 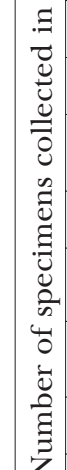 } & $\hat{N}$ & - & & & or & & R & & & & & & & & & & \\
\hline & v & & & & & & & & & & & & & & & & \\
\hline & బิ) & & 아 & & 아 & - & 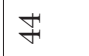 & 10 & & & & & & $\wedge$ & & & H \\
\hline & $\bar{\sigma}$ & & ov & & or & & $\stackrel{\infty}{\sim}$ & & & - & & & & & & & $\tau$ \\
\hline & $\stackrel{\Omega}{=}$ & & F & & on & & $\mathscr{8}$ & & & $\stackrel{\circ}{\circ}$ & & & & - & $\sigma$ & & H \\
\hline & $\stackrel{\infty}{\sim}$ & & & & & & $\exists$ & & & & & & & & & & \\
\hline & $\bumpeq$ & & & & & & & & & & & & & & & & \\
\hline & 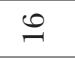 & & - & $H$ & & & $H$ & & 0 & & & & & & & & \\
\hline & 10 & & & & $r$ & & & - & & & & & & & & & \\
\hline & $\stackrel{N}{\sim}$ & & $\infty$ & & & & \& & & & & & & & & & & \\
\hline & $=$ & & & & - & & & & & & & & & & & & \\
\hline & 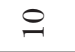 & & & & - & & $\exists$ & & & & & & & & & & \\
\hline & $\infty$ & & & & & & $\bumpeq$ & & & & & & & & & & \\
\hline & 10 & & & & & & & & & & & & & & & & \\
\hline & + & & - & $\infty$ & or & & 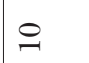 & or & & or & $\infty$ & or & or & or & 10 & $r$ & ov \\
\hline 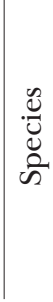 & $\begin{array}{l}\dot{g} \\
\stackrel{a}{0} \\
\dot{a}\end{array}$ & 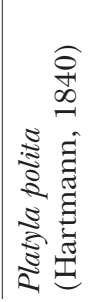 & 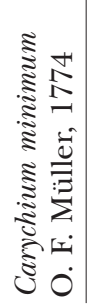 & 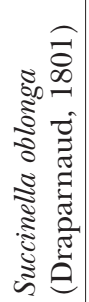 & 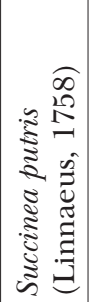 & 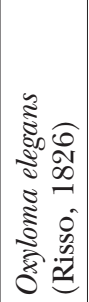 & 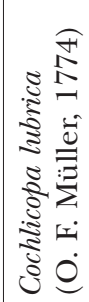 & 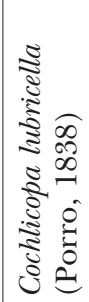 & 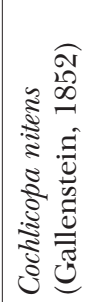 & 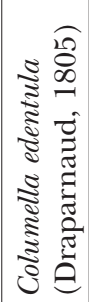 & 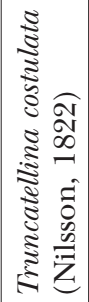 & 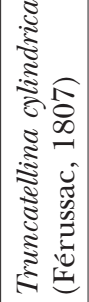 & 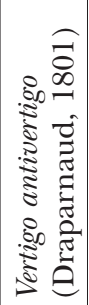 & 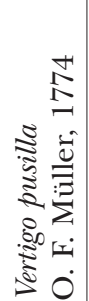 & 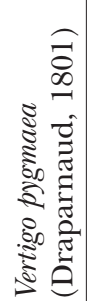 & 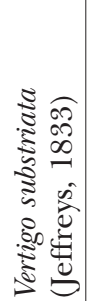 & 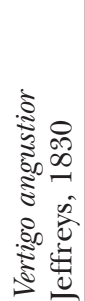 \\
\hline
\end{tabular}




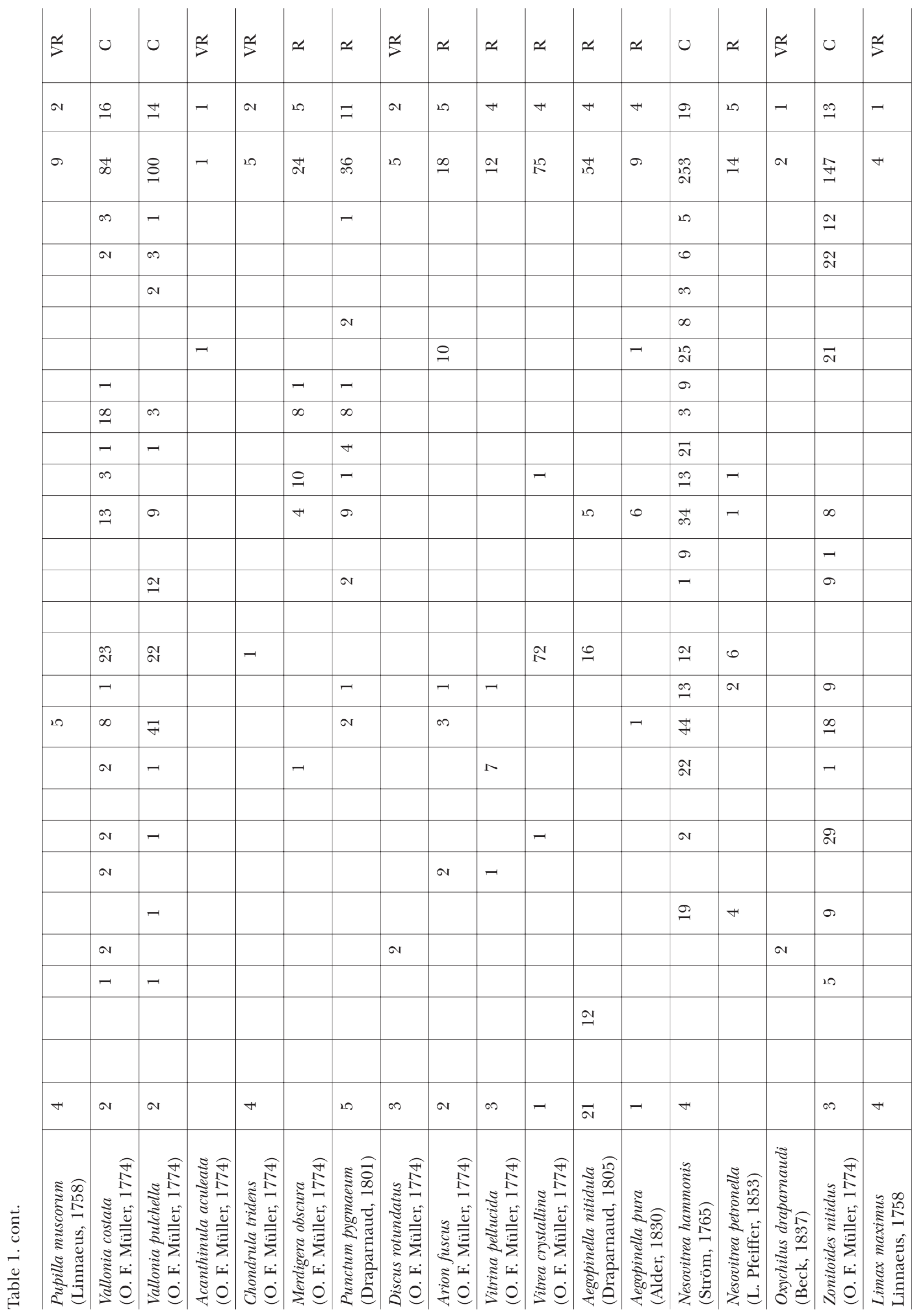




\begin{tabular}{|c|c|c|c|c|c|c|c|c|c|c|c|c|c|c|c|c|c|c|}
\hline 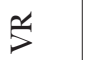 & $\stackrel{f}{s}$ & $\stackrel{f}{s}$ & $\simeq$ & $\stackrel{f}{5}$ & $\stackrel{f}{s}$ & $\simeq$ & $\stackrel{\alpha}{5}$ & $\simeq$ & $\simeq$ & $\simeq$ & U & $\simeq$ & $\simeq$ & $\stackrel{s}{s}$ & $\simeq$ & U & & \\
\hline- & - & or & 0 & $\infty$ & or & 10 & ov & 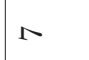 & $\wedge$ & $\sigma$ & $\mathscr{0}$ & $\cong$ & 0 & on & 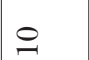 & $\stackrel{g}{\Omega}$ & & \\
\hline \multirow[t]{20}{*}{-} & - & $\mathscr{\sim}$ & $\stackrel{\Omega}{\sim}$ & $\stackrel{\infty}{\underset{\sim}{2}}$ & 10 & $\stackrel{N}{\mathbb{N}}$ & 8 & 8 & จे & 合 & $\stackrel{m}{=}$ & 5 & $\begin{array}{l}\text { \&े } \\
\text { on }\end{array}$ & $\stackrel{\sim}{N}$ & 今ి & YY & $\frac{m}{m}$ & \\
\hline & & & & & & & & & & & & & & & & & के & $\infty$ \\
\hline & & & & & & & & & & $\exists$ & 10 & & & & $\infty$ & & $\pi$ & $\exists$ \\
\hline & & & & & & & & & & & & & & & - & & $\mathrm{N}$ & $H$ \\
\hline & & & & & & & & $\neg$ & & & & & & & & & $\stackrel{\bullet}{\sim}$ & 10 \\
\hline & - & & - & & & -7 & & & ov & & ov & $\neg$ & & & & จ & 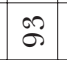 & 2 \\
\hline & & & & & & & & & & & or & $\stackrel{a}{v}$ & & & & 우 & $\infty$ & $\infty$ \\
\hline & & & & & & & & & & - & - & $\neg$ & & & & $\stackrel{-}{\circ}$ & $\mathbb{N}$ & 20 \\
\hline & & & - & & & & & & & & & $H$ & & & & \&ั & $\$$ & $=$ \\
\hline & & & & & & & & - & $H$ & & 10 & 1 & & & & \% & gr & $\exists$ \\
\hline & & & ov & & & & & & 0 & 10 & $\stackrel{\rho}{-}$ & & & & & $\vec{a}$ & I & $\vec{\sigma}$ \\
\hline & & & & & & & & & & & & & & & & & 2 & H \\
\hline & & & & & & & & & - & $\infty$ & & $\neg$ & & & & H & $\stackrel{2}{q}$ & $\exists$ \\
\hline & & & & & & 10 & & & & & & & & & & ov & 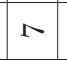 & ov \\
\hline & & & & & 0 & ㅇ & & 年 & - & 0 & 0 & 0 & จิ & $\stackrel{\circ}{\circ}$ & จิ & Â & $\mid \begin{array}{l}\infty \\
\stackrel{\infty}{\alpha}\end{array}$ & $\stackrel{19}{\circ}$ \\
\hline & & & & & & & & $\infty$ & - & 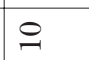 & $\neg$ & o & & & ov & & R & $\stackrel{\infty}{\sim}$ \\
\hline & & $\vec{a}$ & $\theta$ & - & & & L̊ & H & & ov & \& & 0 & 10 & & 8 & \& & $\begin{array}{l}0 \\
0 \\
10\end{array}$ & $\mathscr{\sim}$ \\
\hline & & & on & & & & & 0 & & & & & & & & is & $\stackrel{+}{\stackrel{D}{\sigma}}$ & $\stackrel{\circ}{\sim}$ \\
\hline & & & & & & & & & & & & & & & & 10 & 12 & - \\
\hline & & & & & & & & & & & 0 & & & & & & $\begin{array}{l}\mathbf{I} \\
\end{array}$ & 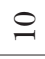 \\
\hline \multirow[t]{7}{*}{-} & & & & & & & & & & & $\infty$ & & $\begin{array}{l}\infty \\
\infty\end{array}$ & & $\stackrel{\varrho}{ٍ}$ & $\hat{0}$ & $\left|\begin{array}{l}\infty \\
\stackrel{n}{a}\end{array}\right|$ & 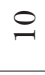 \\
\hline & & & & & & & & & & $\infty$ & & & จ & & $\stackrel{19}{\text { ô }}$ & จิ & $\begin{array}{l}\mathbb{N} \\
\mathbb{N}\end{array}$ & $\stackrel{\circ}{\circ}$ \\
\hline & & & & & & & & & & & $\sigma$ & & จิ & $\mathcal{V}$ & & 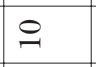 & $\begin{array}{c}\infty \\
10\end{array}$ & $\infty$ \\
\hline & & & & & & & & & & & 10 & & F & & 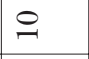 & 10 & $\infty$ & $\sigma$ \\
\hline & & & & - & $\sigma$ & 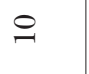 & & & & & $H$ & & 아 & & & $\stackrel{\mathscr{V}}{\sim}$ & $\stackrel{20}{\stackrel{2}{1}}$ & $\infty$ \\
\hline & & & & & & & & & & & or & & If & & 12 & F & $\begin{array}{c}\mathcal{N} \\
\stackrel{2}{2}\end{array}$ & H \\
\hline & & 10 & on & $\exists$ & ô & $\mathscr{0}$ & 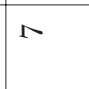 & $H$ & 10 & 0 & 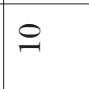 & 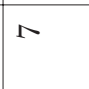 & 1 & $H$ & 1 & 1 & $\begin{array}{l}\text { Oे } \\
\text { ổ }\end{array}$ & 아 \\
\hline 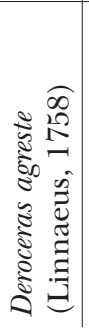 & 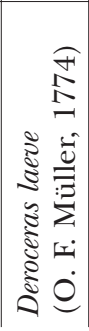 & 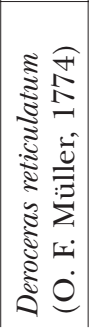 & 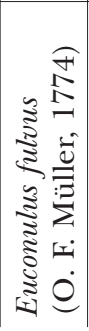 & 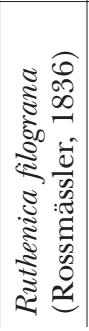 & 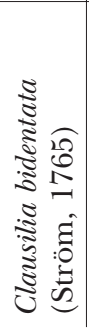 & 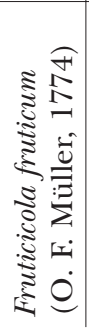 & 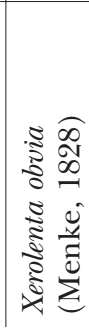 & 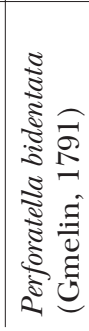 & 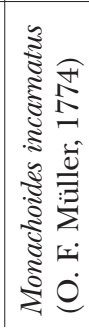 & 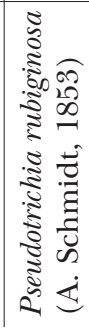 & 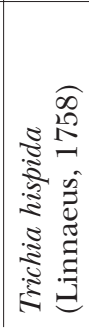 & 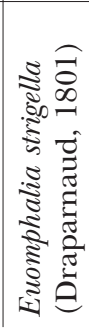 & 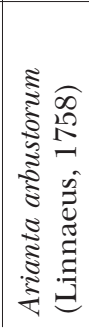 & 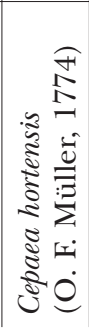 & 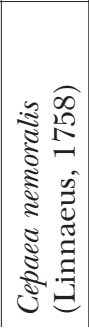 & 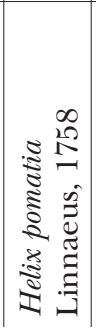 & 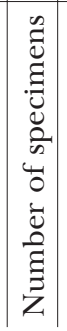 & 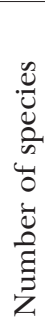 \\
\hline
\end{tabular}


used farmland and harbours several towns (including the city of Poznań). A significant human impact on the ecosystems of the valley has been already observed (GOŁDYN et al. 2005). Our research shows that the valley is important from the malacological point of view, since its relatively small area hosts more than half of the species recorded from the region (even more when the missing species are added). The localities of the rare clausiliid, $R$. filograna, known so far only from seven localities in the Wielkopolska region (KORA-
LEWSKA-BATURA et al. 2010), emphasize the conservation value of the studied area. Also V. angustior, an endangered species from Annex II of the EU Habitats Directive, is present in four plots $(4,19,21,25)$. The species is known from 52 recent localities in the Wielkopolska Region (KORALEWSKA-BATURA et al. 2010). All the areas where $V$. angustior was found during the present study are well-preserved riverine habitats (wet meadows and sedge stands with some riparian forests) typical for the species (PIECHOCKI \& SUlikow-

Table 2. Zoocenological analysis of the terrestrial malacofauna of the Cybina Valley

\begin{tabular}{|c|c|}
\hline Dominance $(\%)$ & Constancy (\%) \\
\hline D5 - eudominants & C5 - euconstants \\
\hline none & Nesovitrea hammonis - $57.58 \%$ \\
\hline D4 - dominants & C4 - constants \\
\hline \multirow[t]{5}{*}{ none } & Cochlicopa lubrica - 48.48\% \\
\hline & Helix pomatia $-43.94 \%$ \\
\hline & Trichia hispida - 36.36\% \\
\hline & Vallonia costata - $33.33 \%$ \\
\hline & Vallonia pulchella - 33.33\% \\
\hline D3 - subdominants & C3 subconstants \\
\hline Helix pomatia $-13.84 \%$ & Carychium minimum - $28.79 \%$ \\
\hline Arianta arbustorum $-10.30 \%$ & Zonitoides nitidus - $28.79 \%$ \\
\hline Cochlicopa lubrica $-8.08 \%$ & Euomphalia strigella - $21.21 \%$ \\
\hline Cepaea nemoralis - $7.92 \%$ & Cepaea nemoralis - $19.70 \%$ \\
\hline \multirow[t]{7}{*}{ Nesovitrea hammonis - $7.92 \%$} & Cochlicopa lubricella - $19.70 \%$ \\
\hline & Punctum pygmaeum - $19.70 \%$ \\
\hline & Arianta arbustorum - $16.67 \%$ \\
\hline & Monachoides incarnatus - $16.67 \%$ \\
\hline & Perforatella rubiginosa - $16.67 \%$ \\
\hline & Perforatella bidentata $-15.15 \%$ \\
\hline & Succinea putris $-15.15 \%$ \\
\hline $\mathrm{D} 2$ - recedents & C2 - accessory species \\
\hline Ruthenica filograna $-4.48 \%$ & Euconulus fulvus - $13.64 \%$ \\
\hline Zonitoides nitidus $-4.60 \%$ & Merdigera obscura $-10.61 \%$ \\
\hline Trichia hispida $-3.54 \%$ & Succinea oblonga - $10.61 \%$ \\
\hline Carychium minimum - $3.51 \%$ & Arion fuscus $-9.09 \%$ \\
\hline \multirow[t]{8}{*}{ Vallonia pulchella $-3.13 \%$} & Nesovitrea petronella $-7.58 \%$ \\
\hline & Vertigo pusilla - 7.58\% \\
\hline & Aegopinella nitidula $-6.06 \%$ \\
\hline & Aegopinella pura $-6.06 \%$ \\
\hline & Truncatellina costulata $-6.06 \%$ \\
\hline & Vertigo angustior $-6.06 \%$ \\
\hline & Vitrea crystallina $-6.06 \%$ \\
\hline & Vitrina pellucida $-6.06 \%$ \\
\hline D1 - subrecedents & C1 - accidental species \\
\hline Remaining 41 species & Remaining 22 species \\
\hline
\end{tabular}




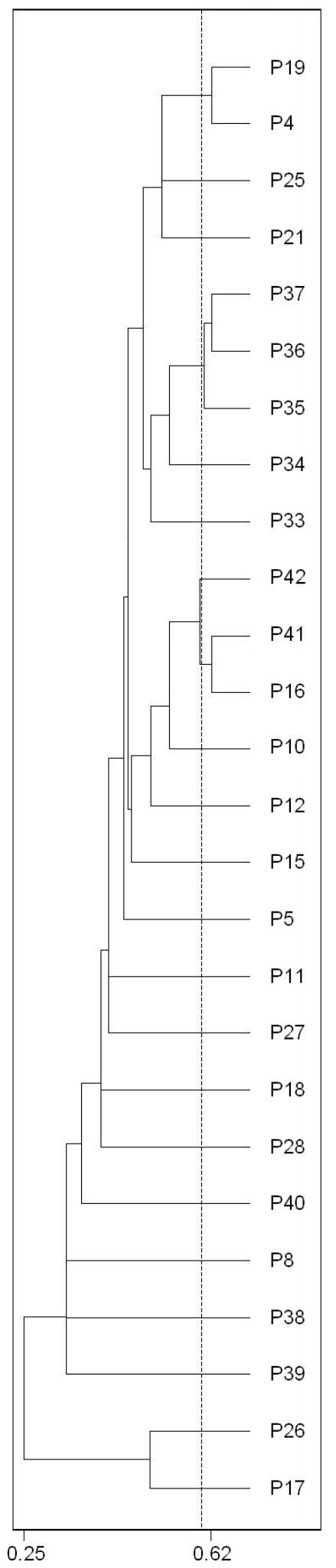

Fig. 2. Similarities in the species composition among the study areas (Marczewski-Steinhaus index)
SKA-DROZD 2008). In all the areas V. angustior was accompanied by C. minimum, S. putris, C. lubrica, V. costata, N. hammonis, P. bidentata, P. rubiginosa, T. hispida, E. strigella and C. nemoralis.

Besides $V$. angustior, included as endangered in the Red List of Threatened Animals in Poland (WIKTOR \& RIEDEL 2002), three species found during the present study are red-listed as near threatened: $N$. petronella (rare in the Cybina Valley, recorded from five plots), T. costulata (rare, four plots) and C. tridens (very rare, two plots). Among the other species that were very rare in the studied area, three ( $C$. bidentata, $C$. nitens and $R$. filograna) are classified as near threatened by PIECHOCKI \& SULIKOWSKA-DROZD (2008), although among them only $R$. filograna is actually rare in the region; there are 66 recent localities of C. bidentata and 76 of C. nitens known in the Wielkopolska region (KORALEWSKA-BATURA et al. 2010).

Of the remaining 17 species classified as very rare in the Cybina Valley, only two are relatively rare in Wielkopolska. P. polita is found in 14 recent localities and, according to WIKTOR (2004), it occurs in the whole of Poland, but its populations are isolated. $O$. draparnaudi, recorded from 17 localities in the region during the last 50 years, is an alien species in Poland, now expanding its range (PIECHOCKI \& SULIKOWSKA-DROZD 2008). Other species, very rare in the present study, are otherwise common or very common in Wielkopolska (e.g. P. muscorum, with 274 localities in the region or $O$. elegans, with 220 localities) and their rarity in the collected material results most likely from the small number of samples, not fully reflecting the diversity of habitats in the Cybina Valley.

Particular sections of the river valley differ in their gastropod species richness. The uneven sampling effort in different plots and the incompleteness of the inventory certainly affect the results. Still, some relations between the species richness and the habitat diversity are obvious. The plots with the greatest mollusc diversity (4, 19, 25 and 33) hold wet habitats, mainly patches of riverine forest and meadows. In the complex evaluation of the nature conservation status of the Cybina Valley (GOŁDYN et al. 2005), these four plots are of high or very high conservation value. Moreover, plot 19 is classified as "outstanding" because of the high value of protected habitats. Plot 4 , where the species richness is the greatest and where four species of high conservation value occur (V. angustior, T. costulata, C. tridens and R. filograna), seems to be the most extraordinary. It is located within the borders of the city of Poznan and holds riverine forests and wetlands moderately transformed under human impact and partly converted into ruderal habitats. The result is a mosaic of habitats suitable for different snail species. The plots with the smallest species richness are almost always covered by dry, monotonous and much human-transformed hab- 
itats: arable fields, meadows, recreational areas or deforested valley slopes.

Our results supplement the data reported in the monograph of the nature of the Cybina Valley (GOŁDYN et al. 2005). As a result of the present study, the number of mollusc-rich plots has increased, and the conservation value of some parts of the valley has turned out to be higher compared to the earlier estimations. The Cybina catchment area or at least its more valuable parts should be legally protected. At present the Cybina Valley is included in the shadow list of the Polish Natura 2000 network.

\section{REFERENCES}

BŁosZYK J. 1999. Geograficzne i ekologiczne zróżnicowanie zgrupowań roztoczy z kohorty Uropodina (Acari: Mesostigmata) w Polsce. I. Uropodina lasów grądowych (Carpinion betuli). Kontekst, Poznań.

BŁoszYK J., Krysiak D., NAPIERAŁA A., DYLEWSKA M. 2006. Can soil fauna undergo synantropisation. In: GABRYŚ G., IgNATOWICZ S. (eds), Advances in Polish Acarology. SGGW, Warszawa, pp. 26-37.

Cameron R. A. D., Pokryszko B. M. 2005. Estimating the species richness and composition of land mollusc communities: problems, consequences and practical advice. J. Conch. 38: 529-547.

Everitt B. S., LANDAU S., LeESE M. 2001. Cluster Analysis. Arnold, London.

GOŁDYN R., GRABIA J. 1998. Program ochrony wód rzeki Cybiny. Urząd Miasta Poznania, Wydział Ochrony Środowiska, Poznań.

GOŁDYN R., JACKOWIAK B., BŁOsZYK J. (eds). 2005. Natural values of the Cybina Valley and their protection. Kontekst, Poznań.

Holgate P. 1969. Notes on the Marczewski-Steinhaus coefficients of similarity. Stat. Ecol. 3: 181-193.

KorAlEWSKA-BATURA E. 1992. Mięczaki (Mollusca) Wielkopolski. UAM Poznań, Zoologia, Poznań 18: 1-38.

\section{ACKNOWLEDGEMENTS}

We thank Mr. COEN MEJER, director of Zeelandia Sp. z o.o. from Tarnowo Podgórne, for his unceasing interest in the project of the Cybina Valley conservation and for the financial support of the present study. Special thanks go to Dr. MICHAє KUPCZYK for his help during the field work. We are very grateful to Dr. JERZY PTASZYK, the Voivodeship Nature Conservator in Poznań, for his continuous interest and support. The research was partially supported by the MNiSW grant no. N N304 340033.

KorAlEwSKA-BATURA E., GOŁDYN B., SZYBIAK K., BŁOSZYK J. 2010. Materials to the knowledge of molluscs of Wielkopolska. II. Checklist. Folia Malacol. 18: 29-41.

Piechocki A., Sulikowska-Drozd A. 2008. Mięczaki (Mollusca). In: Bogdanowicz W., CHudzickA E., PILIPIUK I., SKIBIŃSKA E. (eds.). Fauna of Poland - characteristics and checklist of species. Muzeum i Instytut Zoologii PAN, Warszawa, 3, pp. 365-426.

UNO 1992. Convention on Biological Diversity. Unated Nations Conference on Environment and Development, Rio de Janeiro, June, 3-14 1992.

WikTor A. 2004. Ślimaki lądowe Polski. Mantis, Olsztyn.

WikTOR A., RIEDEL A. 2002. Gastropoda terrestria, Terrestrial gastropods. In: GŁOWACIŃSKI Z. (ed.). Red List of Threatened Animals in Poland. Instytut Ochrony Przyrody PAN, Kraków, pp. 27-33.

WOJTERSKi T., WOJTERSKA H., WOJTERSKA M. 1978. Potencjalna roślinność naturalna środkowej Wielkopolski mapa barwna. PPWK, Wrocław.

Received: January 22nd, 2009

Revised: September 24th, 2009

Accepted: October 25th, 2009 